\title{
Visualization and Evaluation of Prostate Needle Biopsy
}

\author{
Jianchao Zeng, Charles Kaplan', John Bauer ${ }^{2}$, Isabell Sesterhenn ${ }^{3}$, Judd Moul ${ }^{4}$ and Seong K. Mun \\ Imaging Science and Information Systems Center \\ Department of Radiology, Georgetown University Medical Center \\ 2115 Wisconsin Avenue, NW, Suite 603, Washington, DC 20007
}

\begin{abstract}
Three-dimensional (3-D) computer visualization is playing an increasingly important role in medical imaging applications. Visualization is helpful and important because it provides more flexibility for medical education, training and pre-operative planning, and it offers a better method to evaluate schemes currently used in the clinical environment for their improvements and optimizations. We have developed a 3D visualization system for prostate needle biopsy simulation. Surface models of the prostate are reconstructed from the digitized pathological tissue images of the real prostate specimens. The needle biopsy of the prostate can be visualized and simulated automatically by the system or interactively by a urologist with a 6 degreeof-freedom (DOF) tracking device. This system has been used to validate the effectiveness of the automatic simulation for the evaluation of prostate needle biopsy. It has also been used to compare the performance of the existing biopsy schemes. In this paper, further experiments are conducted using this visualization system with a total of 107 reconstructed 3-D prostate surface models to evaluate the correlation between tumor volume and positive needle core volume. Preliminary experimental results are also given.
\end{abstract}

\section{Introduction}

The screening methods of prostate cancer include prostate specific antigen (PSA) and digital rectal exam (DRE). Based on the screening results, a transrectal ultrasound (TRUS) guided prostate needle biopsy may be recommended which currently is the gold standard for the diagnosis of prostate cancer. Due to the low resolution of the ultrasound images, however, a urologist can hardly differentiate abnormal tissues from normal ones during the biopsy. Therefore a number of schemes have been developed to help urologists in doing the prostate needle biopsy, such as the systematic sextant biopsy [Hodge et al. 1989] and the 5-region [Eskew et al. 1997], which designate locations of needles on the prostate as well as number of needles to use. It is reported that the current biopsy schemes need to be improved in terms of their accuracy in cancer detection [Bankhead 1997]. In addition, since tumor volume plays an extremely important role in helping a physician decide which method to use for the cancer treatment, it is necessary to develop a practical approach for the physician to estimate tumor volume from the positive needle core volumes of the biopsy results. Currently there is no accurate approach available used in the clinical environment.

We have developed a 3-D visualization system that can be used to help physicians solve these problems in prostate needle biopsy. Surface models of the prostate are reconstructed from the digitized pathological tissue images of the real prostate specimens. The needle biopsy of the prostate can be visualized and simulated automatically by the system or interactively by a urologist with a 6 DOF tracking device. Experiments have been conducted using the visualization system to show that the automatic simulation is reliable to be used for the evaluation of prostate needle biopsy by comparing its performance to that of a urologist [Zeng, Kaplan et al. 1998]. This system has also been used to evaluate the existing sextant and 5-region biopsy schemes, and it is shown that the 5-region scheme is performing better than the sextant scheme in terms of the rate of tumor detection, and the amount of positive core volume. In this paper, we conduct further experiments with this system to investigate the correlation between the tumor volume and the positive needle core volume. In section 2, the visualization system is briefly described, followed by the evaluation experiments and the preliminary experimental results in section 3 . Conclusions are given in section 4.

\section{The 3-D Visualization System}

\subsection{Reconstruction of the 3-D Prostate Surface Models}

As the first step of developing a visualization system for the prostate needle biopsy. 3-D prostate surface models are constructed from the real specimens of prostates with localized cancer, which were resected from the patients and sliced at $4 \mu \mathrm{m}$ thickness and $2.25 \mathrm{~mm}$ intervals. Each slice was then filmed and digitized with a scanning resolution of 1,500 dots per inch, and the contours of key pathological structures, such as urethra and the seminal vesicle as well as the tumors, were extracted by a pathologist. In order to realize real-time visualization performance, surface modeling is used in the model reconstruction process, which is sufficient for the purpose of visualization of prostate needle biopsy. For each structure, the contours are stacked up and interpolation between each pair of contours is performed using a 3-D elastic model-based technique. The interpolation between adjacent contours $\mathrm{C} 1$ and $\mathrm{C} 2$ is completed by generating a force field that acts on $\mathrm{Cl}$ and forces it to gradually move and conform to $\mathrm{C} 2$. The

\footnotetext{
${ }^{1}$ Urology Division, (jeorgetown University Medical Center, Washington. DC 20007

${ }^{2}$ Department of Urology, Walter Reed Army Medical Center, Washington, DC 20307

${ }^{3}$ Department of Genitourinary Pathology, Armed Forces Institute of Pathology, Washington, DC 20306

${ }^{4}$ The Center for Prostate Disease Research, Uniformed Services University of the Health Sciences, MD 20814-4799
} 
volumes of the tumor and the prostate gland are calculated based on each pair of contours of the structures (tumor and prostate gland) after interpolation, which are important parameters for later evaluation experiments. The 3-D model of each structure in the prostate is finally constructed by tiling triangular patches onto the interpolated contours using a deformable surface-spine model which uses a second order partial differential equation to control the deformation of the surface [Xuan et al. 1997]. After a 3-D model is constructed for each structure, a complete 3D prostate surface model is then constructed by combining the models of all the structures in the prostate. Currently, more than 200 digitized prostate specimens have been acquired, and more than $1003-\mathrm{D}$ prostate surface models have been constructed on an SGI Onyx Infinite Reality 10000 Workstation.

\subsection{Visualization of the Prostate Biopsy}

The visualization system is developed using $\mathrm{C}++$ and the object-oriented 3-D visualization development toolkit Open-Inventor on the SGI Onyx Workstation. Graphical user interface is realized based on the Motif toolkit. While menu operations are mostly performed using a two-dimensional (2-D) mouse, the interactive biopsy simulation is mainly carried out using a 6 DOF tracking device (6-D mouse) which is especially integrated in the visualization system. In addition to general visualization functions, such as model manipulation (e.g., rotation, translation and zooming) and model property change (e.g., transparency and color), this system primarily provides functions specific for the prostate needle biopsy. It has two simulation modes: an automatic simulation and an interactive simulation. The whole process of a prostate needle biopsy with any specific scheme can be simulated based on the reconstructed 3-D prostate surface models. In the automatic simulation mode, the locations for needle insertion on the surface of the prostate are calculated automatically by the computer based on the requirement of the specific biopsy scheme. Thirty degrees of angle with respect to the local normal vector of the prostate surface are also calculated automatically for each needle. Needles are then mounted to the positions in the calculated poses. After shooting the needles, the system then detects which needles are hitting the tumors inside the prostate by calculating ray intersection along the needle direction with the tumors. If there is any (the biopsy is then called positive), the system calculates the positive needle core volumes by the amount of intersection and displays the results on the screen. Since this whole process is controlled by the system, it can be finished very quickly, making it possible to apply this simulation to a large number of samples (3-D prostate models) for later statistical analysis if its performance can be validated. Each step of the automatic biopsy simulation process can also be visualized from any perspective by manipulating the 3-D prostate model in real time. Figure 1 shows the needle locations on the prostate for the sextant and 5-region schemes. Figure 2 shows the needles mounted in these locations in their initial poses. Figure 3 shows the side view of the needles after being fired in the prostate. An example of needle biopsy results for both the sextant and 5-region schemes is shown in Figure 4, where the prostate is displayed in semi-transparency for see-through purpose.

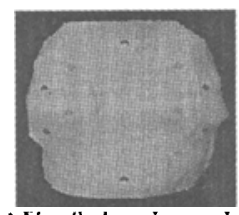

Figure I Needle locations calculated for the sextant and 5-region schemes

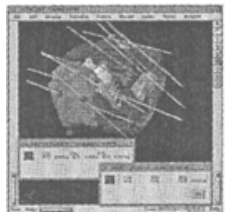

Figure 4 An example of needle biopsy result for sextant and 5-region

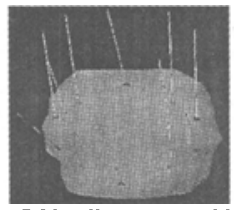

Figure 2 Needles mounted in their initial locations and poses

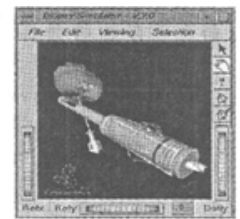

Figure 5 Virtual ultra
and the needle in use

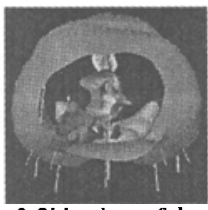

Figure 3 Side view of the needles after being fired in the prostate

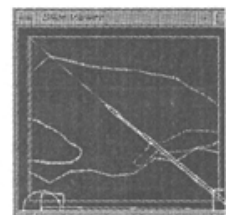

Figure 6 Synthesized ultrasound image with needle path and fired needle

For the interactive simulation, a 6 DOF tracking device is integrated to simulate the ultrasound probe used during actual prostate biopsy procedure. The tracking device consists of an ultrasound transmitter, a controller, and a freely movable receiver device that serves as a tracker. With this device, the system can track both the position ( $x, y$, z) and the orientation angles (Pitch, Yaw, Roll) of the receiver in real time $(50 \mathrm{~Hz})$. The tracking information is simultaneously used in controlling movement of the virtual ultrasound probe in the visualization system. The synthesized ultrasound images are refreshed in real time to follow the movement of the probe. The ultrasound images show intersectional anatomical slices of the prostate as biopsy guidance for the user (a urologist). With this interactive simulation mode, the urologist can perform a virtual needle biopsy as though he/she is performing a real biopsy on a patient. He/she determines the location for each needle insertion based on a specific biopsy scheme under the guidance of the synthesized ultrasound image. The angle of the needle is fixed with the ultrasound probe, 
and the upcoming path of the needle is always displayed and overlaid on the ultrasound image so that the urologist knows where the needle will go through inside the prostate. The result of a biopsy is automatically calculated by the system after each biopsy and is displayed to tell the urologist whether the biopsy is positive or negative and how much the positive needle core volume is. Figure 5 shows the virtual ultrasound probe and the needle in use, while Figure 6 shows the corresponding synthesized ultrasound image with needle path and the fired needle.

\section{Evaluation and Experiments: Correlation between Tumor Volume and Positive Core Volume}

Tumor volume is one of the key parameters to be used for the determination of cancer stage and therapy methods [Tanagho and McAninch 1995]. For example, the therapy for patients with low-stage prostate cancer is currently radical prostatectomy or radiation therapy, while patients with locally extensive cancer are advised to have radiation therapy and surgery is not recommended. It is therefore important for a physician to be able to estimate the tumor volume as accurately as possible, especially when the tumor is still small and non-palpable. Currently there is no practical approach that can help a physician estimate the tumor volume. It is even not sure if it is possible to have such an approach. In this paper, we investigate this possibility by first considering the correlation of the tumor volume to the positive needle core volume. If there is a strong correlation, it suggests that there may be a possibility to estimate the tumor volume from the positive needle core volume. We also investigate the correlation in terms of the PSA values and the race to see if the correlation shows any special features for these special groups of patient.

\subsection{Correlation in General Cases}

We use 107 3-D prostate models on the needle biopsy visualization system with both sextant and the 5-region. Figure 7 shows a correlation result of tumor volume vs. positive core volume with the sextant biopsy scheme. Table 1 gives the corresponding correlation coefficient and the level of significance. Since the level of significance is 0 which is smaller than 0.01 , the correlation is significant between the two volume variables with the sextant scheme. The results with the 5-region scheme are shown in Figure 8 and Table 2, which also indicates that the correlation is significant.

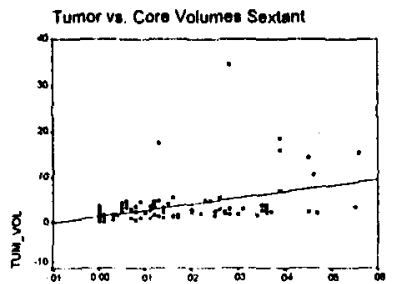

COAE,SHA

Figure 7 Plot of tumor volume vs. core volume with sextant scheme

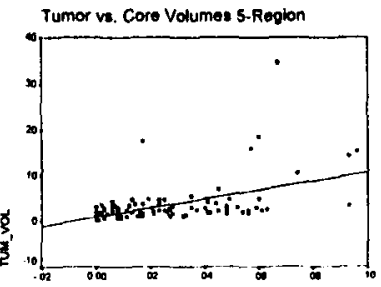

CORE_SAE

Figure 8 Plot of tumor volume vs. core volume with 5 -region scheme

Table 1 Correlation coefficient and the level of significance with sextant scheme

(Tumor volume not normalized)

Correlations of Tumor ve. Core Volumes Sextant

\begin{tabular}{|c|c|c|c|}
\hline \multicolumn{2}{|c|}{ 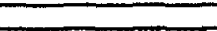 } & CORE STA & TUMM VOL \\
\hline PBद्रुण & CORE_STA & 1.000 & $.440^{\circ}$ \\
\hline Correlation & TUM_VOL & $440^{\circ}$ & 1.000 \\
\hline (2-tailed) & $\begin{array}{l}\text { CORE_STA } \\
\text { TUM VOL }\end{array}$ & 000 & .000 \\
\hline $\mathrm{N}$ & CORE STA & 907 & 107 \\
\hline & TUM_VOL & 107 & 107 \\
\hline
\end{tabular}

(2-tailed).
Table 2 Correlation coefficient and the level of significance with 5-region scheme

(Tumor volume not normalized)

Correlatione of Tumor ve. Core Volumes 6-Region

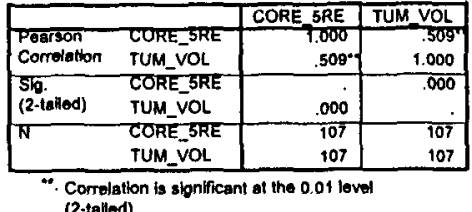

Volume of a prostate gland may also need to be considered since it can affect on capsular penetration which is also a key factor in determining methods for therapy. A tumor may still be confined to a larger prostate gland while another tumor of the same size has already penetrated the capsule of a smaller prostate gland. To further validate the correlation between the tumor volume and the core volume, we first normalize the tumor volume by the volume of prostate gland, and then investigate its correlation to the positive needle core volume for both the sextant and 5region schemes. The results are shown in Figure 9 and Table 3, and Figure 10 and Table 4, respectively. 


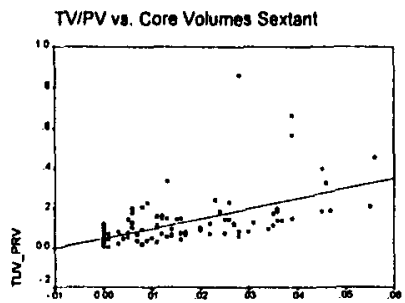

CORE_sTA

Figure 9 Plot of normalized tumor volume vs. core volume with sextant scheme
TVIPV vs. Core Vohumes 5.Region

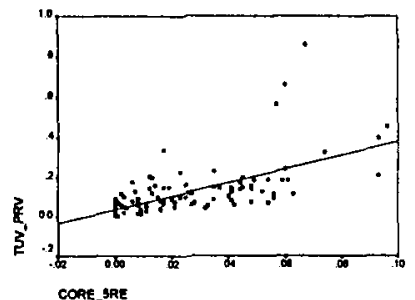

Figure 10 Plot of normalized tumor volume vs. core volume with 5-region scheme
Table 3 Correlation coefficient and the level of significance with sextant scheme

(Normalized tumor volume)

Corrolations

\begin{tabular}{|ll|r|r|}
\hline & & CORE_STA & TUV_PRV \\
\hline Pearson & CORE_STA & 1.000 & $.585^{\circ}$ \\
Correlation & TUV_PRV & $.585^{\circ}$ & 1.000 \\
\hline Sig_. & CORE_STA &. & .000 \\
(2-talled) & TUV_PRV & .000 &. \\
\hline N & CORE_STA & 107 & 107 \\
& TUV_PRV & 107 & 107 \\
\hline
\end{tabular}

". Correlation is significant at the 0.01 lovel (2-talled).
Table 4 Correlation coefficient and the level of significance with 5-region scheme (Normalized tumor volume)

Correlations

\begin{tabular}{|c|c|c|c|}
\hline & & CORE_5RE & TUVPRV \\
\hline $\begin{array}{l}\text { Pearson } \\
\text { Correlation }\end{array}$ & $\begin{array}{l}\text { CORE_STE } \\
\text { TUV PRV }\end{array}$ & $\begin{array}{r}7.000 \\
635^{\circ}\end{array}$ & $\begin{array}{l}.635 \\
1.000\end{array}$ \\
\hline $\begin{array}{l}\text { Shg. } \\
\text { (2-talled) }\end{array}$ & $\begin{array}{l}\text { CORE_5RE } \\
\text { TUV_PRV }\end{array}$ & .000 &.$\overline{000}$ \\
\hline$N$ & $\begin{array}{l}\text { CORE_5RE } \\
\text { TUV_PRV }\end{array}$ & $\begin{array}{l}107 \\
107\end{array}$ & $\begin{array}{l}107 \\
107\end{array}$ \\
\hline
\end{tabular}

As can be seen, the results show a stronger correlation between the normalized tumor volume and the positive needle core volume. The correlation coefficients increase by 0.145 and 0.126 for the sextant and 5-region schemes, respectively. Therefore it can be said that the normalized tumor volume (by the volume of prostate gland) shows a better association with the positive needle core volume than the absolute tumor volume alone.

\subsection{Correlation by PSA Values}

The elevated PSA value is a very important marker to indicate suspicion of prostate cancer. Therefore it may be reasonable to expect that the higher the PSA value the stronger the correlation between the tumor volume and the positive needle core volume. To investigate this possibility, we segment the PSA values into groups and calculate the correlation for each group separately.

\section{(1) Group 1: PSA Values 0-4}

The sample size (number of 3-D prostate models) for this group is 16. The results for both sextant and 5-region schemes are shown in Figure 11 and Table 5, and Figure 12 and Table 6, respectively.

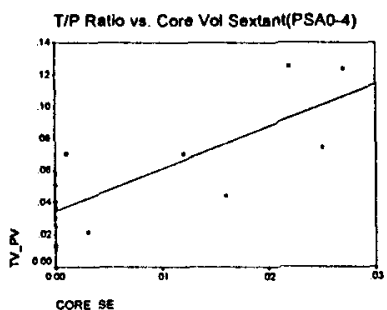

Figure 11 Plot of normalized tumor volume vs. core volume with sextant scheme (PSA0-4)

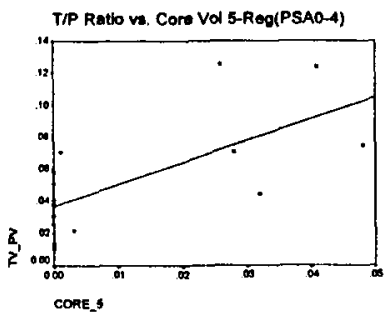

Figure 12 Plot of normalized tumor volume vs. core volume with 5-region scheme (PSA0-4) 
Table 5 Correlation coefficient and the level of significance with sextant scheme (PSA0-4)

(Normalized tumor volume)

Corrolations

\begin{tabular}{|c|c|c|c|}
\hline & & CORE_SE & TVPV \\
\hline $\begin{array}{l}\text { Pearson } \\
\text { Correlation }\end{array}$ & $\begin{array}{l}\text { CORE_SE } \\
\text { TV_PV }\end{array}$ & $\begin{array}{r}1.000 \\
.788^{\circ}\end{array}$ & $\begin{array}{r}7884 \\
1.000\end{array}$ \\
\hline $\begin{array}{l}5 \text { (2-tailed) } \\
\text { (2) }\end{array}$ & $\begin{array}{l}\text { CORE_SE } \\
\text { TV_PV }\end{array}$ & .000 & .000 \\
\hline$N$ & $\begin{array}{l}\text { CORE_SE } \\
\text { TV_PV }\end{array}$ & $\begin{array}{l}16-1 \\
16\end{array}$ & $\begin{array}{l}16 \\
16 \\
\end{array}$ \\
\hline
\end{tabular}

Table 6 Correlation coefficient and the level of significance with 5-region scheme (PSA0-4)

(Normalized tumor volume)

Corrolatione

\begin{tabular}{|c|c|c|c|}
\hline & & CORE 5 & IVPV \\
\hline $\begin{array}{l}\text { Pearson } \\
\text { Correlation }\end{array}$ & $\begin{array}{l}\text { CORE_5 } \\
\text { TV_PV }\end{array}$ & $\begin{array}{r}1.000 \\
.685\end{array}$ & $\begin{array}{r}.685 \\
1.000\end{array}$ \\
\hline $\begin{array}{l}\text { Sig. } \\
\text { (2-tailed) }\end{array}$ & $\begin{array}{l}\text { CORE_5 } \\
\text { TV_PV }\end{array}$ & .003 & .003 \\
\hline 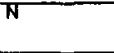 & $\begin{array}{l}\text { CORE_5 } \\
\text { TV_PV }\end{array}$ & $\begin{array}{l}1 \overline{6} \\
16\end{array}$ & $\begin{array}{l}16 \\
16\end{array}$ \\
\hline
\end{tabular}

(2) Group 2: PSA Values 4-10

The sample size for this group is 64 . The results for both sextant and 5-region schemes are shown in Figure 13 and Table 7 , and Figure 14 and Table 8, respectively.

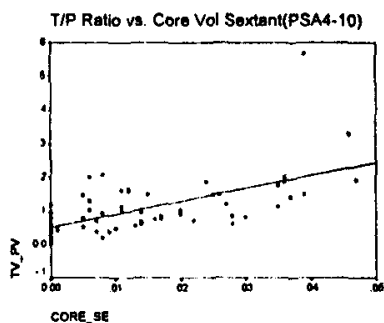

Figure 13 Plot of normalized tumor volume vs. core volume with sextant scheme (PSA4-10)

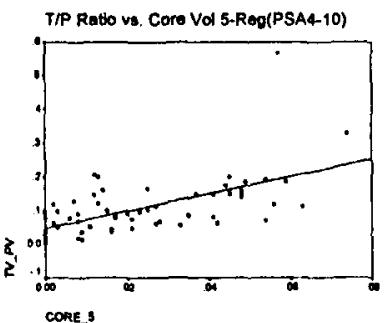

Figure 14 Plot of normalized tumor volume vs. core volume with 5-region scheme (PSA4-10)
Table 7 Correlation coefficient and the level of significance with sextant scheme (PSA4-10)

(Normalized tumor volume)

Correlations

\begin{tabular}{|ll|r|r|}
\hline & & CORE_SE & TV PV \\
\hline Pearson & CORE_SE & 7.000 & .607 \\
Corralation & TV_PV & .607 & 1.000 \\
\hline Sig. & CORE_SE &. & .000 \\
(2-lalled) & TV_PV & .000 &. \\
\hline N & CORE_SE & 64 & 64 \\
& TV_PV & 64 & 64 \\
\hline
\end{tabular}

**. Correlation is significant at the 0.01 level (2-tailed).
Table 8 Correlation coefficient and the level of significance with 5-region scheme (PSA4-10) (Normalized tumor volume)

\section{Correlations}

\begin{tabular}{|ll|r|r|}
\hline & & CORE_5 & TV PV \\
\hline Pearson & CORE_5 & 1.000 & .603 \\
Correlation & TV_PV & $.603 *$ & 1.000 \\
\hline Sig. & CORE_5 &. & .000 \\
(2-tailed) & TV_PV & .000 & \\
\hline N & CORE_5 & 64 & 64 \\
& TV_PV & 64 & 64 \\
\hline
\end{tabular}

"*. Correlation is significant at the 0.01 level (2-tailed).

\section{(3) Group 3: PSA Values More Than 10}

The sample size for this group is 27 . The results for both sextant and 5-region schemes are shown in Figure 15 and Table 9, and Figure 16 and Table 10, respectively.

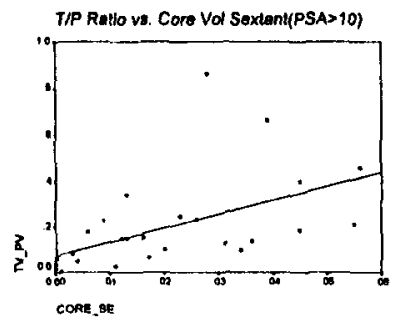

Figure 15 Plot of normalized tumor volume vs. core volume with sextant scheme (PSA>10)

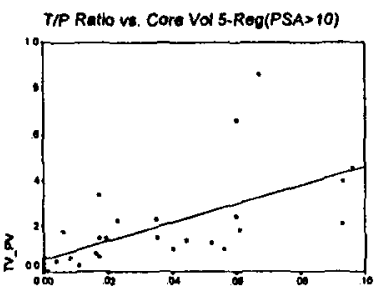

CORE_.

Figure 16 Plot of normalized tumor volume vs. core volume with 5-region scheme (PSA>10) 
Table 9 Correlation coefficient and the level of significance with sextant scheme (PSA>10)

(Normalized tumor volume)

Corrolations

\begin{tabular}{|c|c|c|c|}
\hline & & CORE SE & TVPV \\
\hline $\begin{array}{l}\text { Pearson } \\
\text { Correlation }\end{array}$ & $\begin{array}{l}\text { CORE_SE } \\
\text { TV_PV }\end{array}$ & $\begin{array}{r}1,000 \\
.531^{\circ}\end{array}$ & $\begin{array}{l}.531 \\
1.000\end{array}$ \\
\hline $\begin{array}{l}\text { Sig. } \\
\text { (2-tailed) }\end{array}$ & $\begin{array}{l}\text { CORE_SE } \\
\text { TV_PV }\end{array}$ & .004 & .004 \\
\hline$N$ & $\begin{array}{l}\text { CORE_SE } \\
\text { TV_PV }\end{array}$ & $\begin{array}{l}27 \\
27\end{array}$ & $\begin{array}{l}27 \\
27\end{array}$ \\
\hline
\end{tabular}

Table 10 Correlation coefficient and the level of significance with 5-region scheme (PSA>10) (Normalized tumor volume)

Correlations

\begin{tabular}{|ll|r|r|}
\hline & & CORE_5 & TV_PV \\
\hline Pearson & CORE_5 & 1.000 & .6184 \\
Correlation & TV_PV & $.618 \cdot$ & 1.000 \\
\hline Sig. & CORE_5 &. & .001 \\
(2-tailed) & TV_PV & .001 &. \\
\hline$N$ & \multicolumn{4}{|c|}{ CORE_5 } & 27 & 27 \\
TV_PV & 27 & 27 \\
\hline \multicolumn{3}{|c|}{ T_. Correlation is significant at the 0.01 level } \\
(2-tailed).
\end{tabular}

Unfortunately, the results are not as they were expected: the correlation coefficients do not increase with the PSA values. However, at the moment we cannot conclude that there is no direct association between the PSA values and the correlation since the sample sizes are not large enough for some of the groups. Further investigations will be conducted in this respect.

\subsection{Correlation by Race}

It is recognized that prostate cancer grows differently in different races. In general, African Americans have more chance to grow prostate cancer than Caucasians. In this respect, we investigate the correlation between the tumor volume and the positive needle core volume among African Americans and Caucasians.

\section{(1) African Americans}

The sample size for the African Americans is 28 . The results for both sextant and 5-region schemes are shown in Figure 17 and Table 11 , and Figure 18 and Table 12 , respectively.

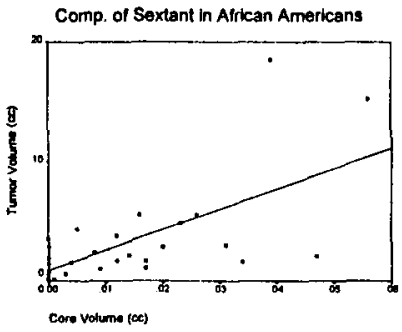

Figure 17 Plot of normalized tumor volume

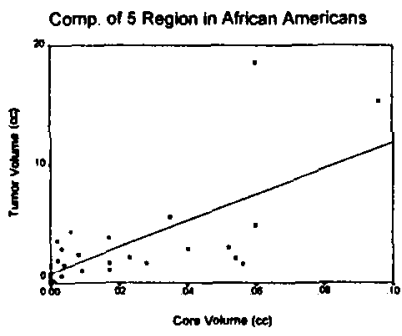

Figure 18 Plot of normalized tumor volume vs. vs. core volume with sextant (African Americans) core volume with 5-region (African Americans)

Table 11 Correlation coefficient and the level of sig. with sextant (African Americans)

\section{Correlatlons}

\begin{tabular}{|ll|r|r|}
\hline & & CORVOL & TUMVOL \\
\hline Pearson & CORVOL & 1.000 & $.643^{\circ}$ \\
Correlation & TUMVOL & $.643^{\circ}$ & 1.000 \\
\hline Sig. & CORVOL &. & .000 \\
(2-tailed) & TUMVOL & .000 &. \\
\hline N & CORVOL & 28 & 28 \\
& TUMVOL & 28 & 28 \\
\hline
\end{tabular}

". Correlation is significant at the 0.01 level (2-tailed).
Table 12 Correlation coefficient and the level of sig. with 5-region (African Americans)

\section{Correlations}

\begin{tabular}{|ll|r|r|}
\hline & & CORVOL & TUMVOL \\
\hline Pearson & CORVOL & 1.000 & .681 \\
Correlation & TUMVOL & .681. & 1.000 \\
\hline Sig. & CORVOL &. & .000 \\
(2-tailed) & TUMVOL & .000 &. \\
\hline $\mathrm{N}$ & CORVOL & 28 & 28 \\
& TUMVOL & 28 & 28 \\
\hline
\end{tabular}

"*. Correlation is significant at the 0.01 level (2-tailed) 
(2) Caucasians

The sample size for Caucasians is 77. The results for both sextant and 5-region schemes are shown in Figure 19 and Table 13, and Figure 20 and Table 14, respectively.

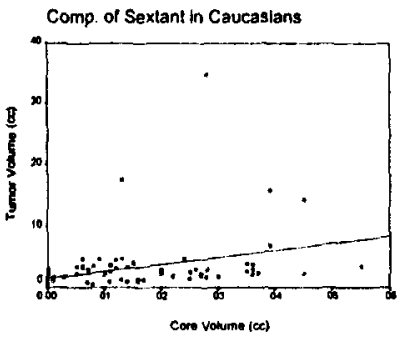

Figure 19 Plot of normalized tumor volume vs, core volume with sextant (Caucasians)

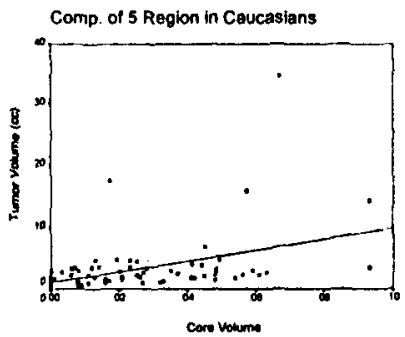

Figure 20 Plot of normalized tumor volume vs. core volume with 5-region (Caucasians)

As expected, the correlation for the African Americans is much stronger than that of the Caucasians. The correlation coefficients increase by 0.295 and 0.250 for the sextant and 5-region schemes, respectively. This may imply that race is an important factor in estimating the tumor volume from the positive needle core volume. Further investigations will be done to confirm this possibility.

\subsection{Summary}

We have experimentally shown that in general there exists a significant correlation between the tumor volume and the positive needle core volume. This correlation is even stronger between the normalized tumor volume (by the volume of prostate gland) and the positive needle core volume. This result supports the possibility that the

Table 13 Correlation coefficient and the level of sig. with sextant (Caucasians)

\section{Correlations}

\begin{tabular}{|ll|r|r|}
\hline & & CORVOL & TUMVOL \\
\hline $\begin{array}{l}\text { Pearson } \\
\text { Correlation }\end{array}$ & CORVOL & 1.000 & .348 \\
\hline Sig. & CORVOL & $.348^{*}$ & 1.000 \\
\hline (2-tailed) & TUMVOL & .002 & .002 \\
\hline $\mathrm{N}$ & CORVOL & 77 & 77 \\
& TUMVOL & 77 & 77 \\
\hline & TU* Correlation is significant at the 0.01 level \\
(2-tailed).
\end{tabular}

Table 14 Correlation coefficient and the level of sig. with 5-region (Caucasians)

\section{Correlations}

\begin{tabular}{|ll|r|r|}
\hline & & CORVOL & TUMVOL \\
\hline Pearson & CORVOL & 1.000 & .437 \\
Correlation & TUMVOL & $.431 *$ & 1.000 \\
\hline Sig. & CORVOL &. & .000 \\
(2-lailed) & TUMVOL & .000 &. \\
\hline N & CORVOL & 77 & 77 \\
& TUMVOL & 77 & 77 \\
\hline
\end{tabular}

". Correlation is significant at the 0.01 level (2-tailed).

larger the core volume the larger the tumor volume. Since there may be other factors, such as locations of the positive needles, that contribute to the prediction of tumor volume, more controlled experiments will be needed to confirm the cause-and-effect relationships between the positive core volume and the tumor volume.

Due to insufficient data, the correlation between the tumor volume and the positive core volume does not increase with the PSA values. However, this correlation does increase for African Americans in comparison to Caucasians.

\section{Conclusions}

We have developed a 3-D computer visualization system, and have conducted experiments with a large number of 3-D prostate models using this system. To explore the possibility of estimating tumor volumes from the positive needle core volumes, we have investigated the possible correlation between the tumor volume and the positive needle core volumes with 107 3-D prostate models. A significant correlation is found between these two kinds of volumes, which supports the possibility that the tumor volume may be reasonably estimated from the positive necdle core volumes. More controlled experiments will be conducted to confirm the cause-and-effect relationships before we will move forward to develop a mathematical model that can predict and estimate the tumor volumes from the positive needle core volumes. In addition, new prostate models will be reconstructed and used to verify the prediction model of tumor volume, and clinical evaluation will also be conducted. 
The 3-D visualization system provides an ideal platform for the simulation and evaluation of prostate needle biopsy. It is also useful for education, especially for visualizing anatomy of prostate, and training of residents and medical students. With the advancement of imaging technologies and improvement of imaging quality, it becomes quite possible to develop an on-line prostate needle biopsy system which provides real time augmented 3-D prostate images to help a urologist to quickly and precisely identify the abnormality in the prostate inside the patient's body. This idea is not limited to the prostate needle biopsy; it may be applied to any type of biopsy, such as kidney biopsy. It may also be applied to other surgeries beyond biopsies, making it possibje to realize a real on-site image-guided minimally-invasive surgery system.

\section{References}

Bankgead, C.: Sextant biopsy helps in prognosis of Pca, but it's not foolproof. Urology Times, Vol. 25, No. 8, August 1997.

Eskew, A. L., Bare, R. L. and McCullough, D. L.: Systematic 5 region prostate biopsy is superior to sextant method for diagnosing carcinoma of the prostate. J. Urol., 157: 199, 1997.

Hodge, K. K, et al.: Random systematic versus directed ultrasound guided trans-rectal core biopsies of the prostate. J. Urol., 142: 71, 1989.

Tanagho, E. A. and McAninch, J. W. (Eds): Smith's General Urology (14 th Edition), Appleton \& Lange, 1995.

Xuan, 3., Hayes, et al.: Surface reconstruction and visualization of the surgical prostate model. SPIE Medical imaging, 1997.

Z.eng, J., Kaplan, C., et al.: Optimizing prostate needle biopsy through 3-D simulation. SPIE Medical Imaging'98. San Diego, February 1998. 